\title{
El emprendimiento desde la perspectiva de las incubadoras de empresas: Un análisis comparado por países
}

\author{
Edwin Moposita-Yanchatuña'; Pilar Rivera-Badillo²; Diana Morales-Urrutia³
}

Fecha de recepción: 18 de Agosto 2018

Fecha de aceptación: 19 de Septiembre 2018

\section{Resumen}

El apoyo al emprendimiento a través de las incubadoras potencia el desarrollo económico de los países. El objetivo de esta investigación es realizar un análisis comparado de la evolución de las incubadoras en siete países (Reino Unido, Alemania, España, México, Brasil, Chile y Ecuador). Para el estudio empírico, se utilizan datos secundarios oficiales de Europa y Latinoamérica, en el periodo 2013-2017. Utilizando la técnica ANOVA se evidencia las diferencias significativas en la creación de las incubadoras y aplicando ANOVA factorial se obtienen tres grupos de países que comparten similitudes en el comportamiento al momento de crear las incubadoras.

Palabras clave: Incubadoras, emprendimiento, países

\section{Abstract}

Support for entrepreneurship through incubators enhances the economic development of countries. The objective of this research is to perform a comparative analysis of the evolution of incubators in seven countries (United Kingdom, Germany, Spain, Mexico, Brazil, Chile and Ecuador). For the empirical study, official secondary data from Europe and Latin America are used in the 2013-2017 period. Using the ANOVA technique reveals the significant differences in the creation of the incubators. Applying factorial ANOVA three groups of countries are obtained that share similarities in the behavior when creating the incubators.

Keywords: Incubators, entrepreneurship, countries

\section{Introducción}

En el presente trabajo se detalla la importancia del emprendimiento para el desarrollo de cada país, es así como, las incubadoras empresariales en el transcurso de los años han tenido un crecimiento importante en la creación de estos centros, desde sus servicios ofertados y espacios físicos, fomentando su réplica en varios países del mundo.

Por lo tanto, el objetivo general de este trabajo es analizar desde un punto de vista comparado, la creación de incubadoras en países de Latinoamérica y Europa. Además, revisar la literatura para construir el marco teórico relacionado con el tema propuesto, del surgimiento del emprendimiento desde la perspectiva de las incubadoras empresariales. Para alcanzar este objetivo, desde el punto de vista metodológico se realiza una combinación de un análisis teórico y aplicado, que parte de una revisión sistematizada de la literatura, así como del uso de técnicas de análisis descriptivo y estadístico. Para ello se hace uso de información estadística procedente de las siguientes bases de datos: Anprotec, Sebrae, Senescyt, Ministerio de Industria y Productividad (MIPRO), Institución de Corfo en Chile y artículos científicos entre los más destacados Blanco, Ferreiro, Perdomo, Risso, Vaquero entre otros. Desde el punto de vista espacial el análisis se centra en siete países: Reino Unido, Alemania, España, México, Brasil, Chile y Ecuador, que fueron seleccionados en base a las disponibilidades estadísticas y porque representan diferentes realidades sociales y económicas, en un período que comprende 2013-2017.

Desde el punto de vista analítico, el presente trabajo se compone de 3 partes, además de la introducción y de las conclusiones. En una primera parte, se realiza una revisión sistematizada de la literatura referencial con el fin de comprender mejor de cómo han surgido las incubadoras empresariales y su evolución en el transcurso del tiempo. Además, se puede comprender la diferencia existente entre incubadora y vivero.
Dentro de esta misma sección se incluye el análisis de la importancia que tienen las incubadoras en la aportación a la economía de un país. En la segunda parte se describe la metodología aplicada para el desarrollo de esta investigación, así como los datos de las bases empleadas y las limitaciones presentadas tanto en cuestiones estadísticas como en el procesamiento de la información. Se incluye además una descripción detallada de las variables objeto de análisis.

Finalmente, en la tercera parte se aborda un estudio empírico aplicado a siete países Reino Unido, Alemania, España, México, Brasil, Chile y Ecuador analizados a través del estadístico Anova, que permitirá observar si existe diferencia u homogeneidad en la creación de incubadoras empresariales en los diferentes países.

\section{Notas históricas}

El emprendimiento es parte fundamental en el desarrollo económico de cada país y ha sido impulsado con mayor fuerza por organismos internacionales, creando de esta manera políticas de crecimiento, a través de nuevos emprendimientos, por consiguiente, nuevas empresas (Messina y Hochsztain, 2015; Perdomo, Arias y Lozada, 2016). La Organización de las Naciones Unidas (ONU) aprobó en la resolución 67/2012, el emprendimiento para el desarrollo, para contribuir con la generación de empleo y desarrollo sustentable (Messina y Hochsztain, 2015): es decir, donde hay un espíritu emprendedor hay una gran probabilidad que existan ideas empresariales que puedan contribuir al crecimiento y desarrollo considerable de la economía (Ferreiro, Del Campo y Camino 2015). Además, ayuda al autoempleo, generación de riqueza, y bienestar social (Valdés, 2015).

Las incubadoras empresariales tienen como objetivo la creación de nuevos negocios, siendo una herramienta canalizadora de procesos de emprendimiento, permitiendo la transferencia tecnológica, a través de espacios físicos que permitan la creación de nuevas empresas Start-up ${ }^{4}$ y Spin-off . La evolución del emprendimiento ha influenciado de igual manera en la aparición de las incubadoras que han diversificado sus servicios hacia la innovación tecnológica (Valdés, 2015).

Las incubadoras o viveros empresariales dinamizan el desarrollo económico, nacional y regional de los países y su impacto es considerable en el crecimiento de los micros y pequeños empresarios en todo el mundo. Es en estas incubadoras empresariales donde se detectan y potencializan las ideas innovadoras a través de asesoramiento especializado en la creación del negocio (Guerra, Hernández y Triviño, 2015). Por tanto, se consideran aliadas de los emprendedores (Vargas, 2014).

Las incubadoras surgen en la década de los 50 en Silicon Valley-California en la Universidad de Stanford, donde se creó un parque industrial y que posteriormente se transforma en un parque tecnológico, con el fin de transferir de las universidades hacia las empresas, el conocimiento desarrollado en esta área (Lahorgue, 2010; Fernández y Blanco, 2011; Alba, 2015; Risso, Díaz y Ramassotti, 2015; Valdés, 2015; Ferreiro, Mendoza y Hernández, 2018).

En la década de los 60 y 70 en Estados Unidos los modelos de incubadoras o viveros se expandieron debido al declive industrial que ocurrió en esa época, así como por las políticas gubernamentales deficientes que incrementaron el desempleo. Estos factores impulsaron a los gobiernos federales a considerar a las incubadoras como una herramienta política industrial de sostenimiento económico y por ende

'Estudiante en Organización de Empresas. Universidad Técnica de Ambato. Ambato-Ecuador, E-Mail: emoposita4861@uta.edu.ec

${ }^{2}$ Doctora PhD en Ciencias de la Empresa. Docente. Facultad de Ciencias Administrativas de la Universidad Técnica de Ambato. Ambato-Ecuador. E-Mail: pl.rivera@uta.edu.ec

${ }^{3}$ Doctora PhD en Desarrollo Económico e Innovación. Docente. Facultad de Ciencias Administrativas de la Universidad Técnica de Ambato. Ambato-Ecuador. E-Mail: dc.moralesu@uta.edu.ec ${ }^{4}$ Empresa de nueva creación, que puede ser escalable más rápida, haciendo uso de tecnologías digitales.

${ }^{5}$ Término anglosajón para referirse a una empresa u organización nacida como extensión de otra mediante la separación de una división subsidiaria. 
de desarrollo (Blanco, Garcia, Fernández, Oliva, Bassa y Vaisman, 2018). No obstante, en la década de los años 70 colapsa la industria tradicional, apareciendo nuevas estrategias para la regeneración de los sectores en crisis y paralelamente la necesidad de proporcionar servicios de asesoramiento empresarial, mayoritariamente sin fines de lucro (Fernández y Blanco, 2011; Alba, 2015).

Viadiu y Martínez (2006) manifiestan que desde entonces los viveros y las incubadoras han ido evolucionando con el tiempo. A finales de 1970 e inicios de 1980, las incubadoras empresariales ofrecían espacios físicos, donde podían realizar sus actividades administrativas en beneficio de los nuevos emprendedores, tales como: asesoramiento en el start-up, actividades financieras, alianzas con otras empresas, entre otras, a través de las cuales se incrementaba el empleo con una repercusión favorable para el crecimiento económico de la región y el país. Esto trascendió en los Estados Unidos y estimuló su réplica a las diferentes localidades, como la creación de programas formales de incubadoras por parte de la Administración para el desarrollo económico ${ }^{6}$. Su acogida fue exitosa en el periodo 1995-2000 llegando a crearse un promedio de una incubadora por semana y en el quinquenio alrededor de 2.000 (Lahorgue, 2010; Risso et al., 2015).

La creación de nuevas empresas Pymes impulsó el desarrollo nacional de varios países y sus gobiernos adoptaron estas iniciativas con el propósito de disminuir la probabilidad de fracaso e incrementar la tasa de creación de nuevas empresas (Alba, 2015).

\section{Evolución en Europa}

Actualmente, es común encontrar programas de incubación en las principales economías del mundo como es el caso de Europa. En los años 70, aparecen en Inglaterra, los primeros centros de iniciativas empresariales, promoviendo el desarrollo económico de las pequeñas empresas y negocios de los emprendedores. British Steel Corporation, fue uno de los promotores de esta idea, con el objetivo de contribuir a la competitividad y crecimiento en la innovación (Fernández y Blanco, 2011; Ferreiro y Camino, 2016; Ferreiro et al., 2018).

Ferreiro et al., (2018) mencionan que en la década de los 80, la comisión europea, en conjunto con las universidades que usaban los viveros para el desarrollo de Spin-off, potenciaron la creación de centros de empresas e innovación que permitían comercializar los productos y servicios generados por sus centros de investigación (p. 5). En este mismo periodo se crea la National Business Incubator Asociation (NBIA), como organismo de fortalecimiento empresarial. Desde 1990 hasta la actualidad, se han desarrollado programas comunitarios, cuya finalidad es eliminar los obstáculos al desarrollo empresarial, la creación y el sostenimiento de las empresas, así como apoyar la capacidad de emprender y generar nuevos empleos, a través de viveros públicos y privados (Vaquero y Ferreiro, 2015).

\section{España}

Los primeros viveros aparecieron en los años 80, para promover el desarrollo local y regional tras la política, económica y administrativa, donde el objetivo de las Agencias de Desarrollo Regional (ADR) y las Corporaciones Locales (CC.LL.), era promover el desarrollo económico y generación de empleo en el país (Vaquero y Ferreiro, 2015). Un dato importante en España respecto a la actividad empresarial que realiza entre el año 2004 a 2010, es que el $20 \%$ de las nuevas empresas creadas desaparecieron en sus primeros años, el 15\% desaparecen en su segundo año, mientras en su tercer año el 10\% de los negocios no sobrevivieron. El restante, 55\% de empresas perduraron durante los tres primeros años, teniendo en cuenta que en los últimos años se han reducido los índices de desaparición de las empresas, debido al apoyo de entidades públicas, mismas que ofertaban variedad de servicios a través de viveros, escuelas de negocios, entre otras, en beneficio de los nuevos negocios. El crecimiento de los viveros empresariales genera un efecto positivo en los nuevos negocios de España, permitiendo a las pequeñas empresas sobrevivir en un 90\% reduciendo así su mortalidad prematura (Blanco et al., 2018).
En España existió un crecimiento empresarial considerable generado por apoyo de los centros de desarrollo y en los últimos años especialmente se evidencia la creación de empresas tipo Pymes. Andalucía es la región que más viveros registró, un total de 234 en el año 2013. Su metodología de crecimiento fue la sistematización en experiencias, que con el pasar del tiempo se creó una red empresarial con los municipios locales ya que no tenían un desarrollo significativo (Valenciano y Uribe, 2016).

Según, Ferreiro y Camino (2016) los viveros empresariales de Galicia en el año 2013 crearon en total 1.044 empresas, siendo los más representativos en estos datos el Centro de Iniciativas Empresariales Tecnópole (Ourense); UNINOVA-USC (Santiago de Compostela) y A Granxa (Vigo), registrando al final de ese mismo año 3.394 puestos de trabajo producto de estos centros en apoyo a nuevos negocios.

\section{Latinoamérica}

La idea de las incubadoras de Europa trasciende hasta América Latina como un apoyo a la falta de conocimiento para emprender y los pocos recursos que el emprendedor posee para poner en marcha su proyecto de negocio, es entonces cuando las universidades potenciaron las ideas de los estudiantes emprendedores, aprovechando de su infraestructura, talento humano, recursos técnicos y tecnológicos (Guerra et al., 2015).

\section{México}

La creación de incubadoras y viveros tienen los componentes de innovación y emprendimiento que se buscan integrar con entidades públicas y privadas. En el año 1990 en México a través del Consejo Nacional de Ciencia y Tecnología (CONACYT) una de las instituciones promotoras para la creación de nuevas incubadoras que cuenta con el apoyo del Programa Nacional de Incubadoras de Empresas de Base Tecnológica (PIEBT) y juntas tenían como fin acompañar a la creación de nuevas empresas y su enfoque estaba en el desarrollo de tecnología. Posteriormente en el año 2003, la secretaría de economía creó el Fondo Pymes que fue gestionado por las incubadoras. En 2009 se registraron 500 incubadoras existentes de las cuales 217 se enfocan en empresas tradicionales, 262 corresponden a tecnología intermedia y 21 de alta tecnología, contribuyendo en la creación de 24.394 fuentes de empleo (Vargas, 2014).

Para el año 2013 surge el Instituto Nacional del Emprendedor (INADEM), entidad que impulsó a incubadoras y emprendedores con una política de apoyo a la innovación y el desarrollo económico, teniendo como resultado un incremento considerable en la creación de Pymes. Según el INEGI ${ }^{7}$ (2012), en 2012 se crearon 1'135.089 empresas nuevas a nivel nacional, de las cuales los sectores más relevantes tienen la siguiente participación: 20,4\% pertenecen a la industria manufacturera, el $28,4 \%$ al sector comercial y el $30,7 \%$ al sector de servicios privados no financieros, finalmente el $20,5 \%$ en otras actividades. Datos que permitieron centrar a las incubadoras como aliadas estratégicas para los emprendimientos (Vargas, 2014).

El gobierno fortaleció a las incubadoras empresariales como eje principal de apoyo a las Pymes, con la finalidad de reducir la mortalidad empresarial que de acuerdo con los registros oficiales entre el $80 \%$ y $93 \%$ de las empresas sin asesoría morían en los próximos 5 años de su creación (Guerra et al., 2015). Con esta iniciativa apoyan a las empresas en su etapa de crecimiento y los índices de mortalidad se reducen al $20 \%$, además según la secretaría de economía el $80 \%$ de estas son exitosas (Vargas, 2014). Para autores como Valdés (2015) las incubadoras tradicionales se han incrementado considerablemente hasta el 2013 en un 292\% a diferencia de las incubadoras tecnológicas intermedias que han crecido en 142,85\%. Además, México registra un incremento en la economía interna del país a través del emprendimiento asociativo con jóvenes y mujeres.

\section{Brasil}

Este país es un referente de Latinoamérica. En 1984 trascendió mediante una transferencia de tecnología de las universidades al sector productivo, creando en el año de 1987 la Asociación Nacional de En- 
tidades Promotoras de Emprendimientos de Tecnologías Avanzadas (ANPROTEC), desde su inicio asistió a la creación de aproximadamente 300 incubadoras (Lahorgue, 2010).

En Brasil, las incubadoras generalmente son creadas por iniciativas de instituciones municipales, universidades, centros estatales de ciencia y tecnología, que apoyan los proyectos innovadores de los emprendedores. En este país el $40 \%$ corresponde al total de las incubadoras de base tecnológica y son operadas en su mayoría por iniciativa de universidades y centros de investigación, que ofrecen espacios adecuados para su actividad, y que, dentro de tres o cuatro años, éstas se conviertan en empresas productivas, que aporten al desarrollo del país y la localidad (Maculan, Jiménez y Castellanos, 2015). Además, se caracteriza por una variedad de acuerdos relacionados con desarrollo tecnológico. Cabe recalcar que en Brasil los emprendimientos son apoyados por el Estado, por ende, a las incubadoras para el asesoramiento a nuevos negocios, creando alianzas entre universidades y las empresas correspondientes a innovación (De Sousa, Goncalves, Almeida, y Sacomano, 2017). Finalmente, obtuvo una variación creciente en su Producto Interno Bruto (PIB) gracias a nuevos emprendimientos, registrando en el año 2012 un incremento del PIB de 4,40\% y en el año 2013 de 4,84\% (Da Silva, Furtado y Zanini, 2015).

\section{Chile}

A finales de los años 90, se crea la primera empresa incubadora EmpreNet apoyada por la Universidad de Chile (Facultad de Ciencias, Física y Matemáticas) que actualmente es conocida como Acces Nova, entidad que impulsó la creación de 3 incubadoras universitarias. Posteriormente a finales del año 2000, se fomenta una nueva línea de financiamiento FDI CORFO, su objetivo era promover la creación de nuevas incubadoras universitarias, generando una red para el desarrollo económico del país, además del incremento de fuentes de empleo y bienestar en la sociedad. Cabe mencionar que en Chile predominan dos tipos de incubadoras, la primera que gestionan las universidades orientadas a proyectos tecnológicos y la segunda, los municipios como apoyo a negocios tradicionales (Valdés, 2015). Registrando en el año 2012 más de 20 incubadoras (Gonzalez, 2017).

\section{Ecuador}

Para algunos países, la generación de las incubadoras empresariales ha constituido la base para el desarrollo y crecimiento de la nación (Guerra et al., 2015). En el caso de Ecuador, la Constitución de la República apoya las iniciativas de creación de incubadoras de empresas con el propósito de asistir a los pequeños negocios o emprendimientos, en un marco de equidad social para mejorar la calidad de vida y construir un sistema económico justo, democrático, productivo, sostenible y solidario.

En la última década se ha detectado poca competitividad e innovación en el tejido empresarial, producto de las débiles relaciones entre universidades y empresas. Es así como las universidades ecuatorianas han cambiado su visión hacia un enfoque de vinculación con la investigación y el sector empresarial (Boza, Tachong y Moreira, 2016). En Ecuador se registraron 40 incubadoras de empresas en el 2015 (Senescyt, 2015), dos años más tarde, la Secretaría de Educación Superior Ciencia, Tecnología e Innovación (SENESCYT) acreditó a 11 incubadoras empresariales relacionadas con las universidades, entre las más importantes en el sector público está ESPOL-TECH E.P. en Guayaquil; y Yachay E.P. en Quito, mientras en el sector privado se encuentra la Fundación para el Desarrollo Social Empresarial-UTPL-PRENDHO en Loja; la Universidad Politécnica Salesiana en Cuenca; y en Ambato CORPOAMBATO. Estas incubadoras brindan asesoramiento y fortalecen a los pequeños negocios, actividades económicas en proceso de creación, con el objeto de reducir la mortalidad de las Pymes ecuatorianas (SENESCYT, 2017).

En agosto de 2017 el Ministerio de Industrias y Productividad (MIPRO) dispuso un espacio de innovación para incubadoras, donde se fomentó el desarrollo de proyectos innovadores para emprendedores, donde su objetivo era dar acompañamiento y asesoramiento desde el inicio de la idea hasta su consolidación como una nueva empresa (MIPRO, 2017). Las incubadoras empresariales ecuatorianas ofrecen servicios jurídicos, asesoramiento financiero y comercial, revitalizando y contribuyendo en el marco de la Economía Popular y Solidaria (EPS) (Boza et al., 2016).

En los últimos años en Ecuador se han formalizado varios negocios nuevos, a pesar de la existencia de trabas burocráticas y administrativas (acceso al crédito, trámites legales, etc.), se registra un aumento de la tasa de emprendimiento con el 26,6\%; a diferencia de Chile que presenta un $23 \%$ y Perú un 20\%. Es decir, en Ecuador del 100\% de emprendimientos que surgen, el $40 \%$ corresponde a la transformación industrial, minería, agrícola y senvicios a empresas, el 60\% corresponde a senvicios de compra y venta, peluquería, alimentos, entre otros (Guerra et al., 2015). Por otra parte, las incubadoras han ido evolucionando desde sus inicios en varias regiones, las mismas que han adoptado como una estrategia de desarrollo económico (Risso et al., 2015), siendo su objetivo promocionar a las nuevas empresas y asistirlas hasta que puedan mantenerse de manera autónoma en el mercado competitivo (Ferreiro et al., 2018).

Tabla 1. Resumen de la evolución de Incubadoras empresariales

\begin{tabular}{|c|c|}
\hline Década & Descripción \\
\hline \multirow{3}{*}{70} & Seminarios dirigidos \\
\hline & Agencias de empresas \\
\hline & Polígonos industriales \\
\hline 80 & Incubadoras de empresas \\
\hline \multirow[b]{2}{*}{90} & $\begin{array}{l}\text { Incubadoras multifuncionales } \\
\text { Incubadoras tecnológicas }\end{array}$ \\
\hline & $\begin{array}{cl}\text { Incubadoras } & \text { Incubadoras sin muros } \\
\text { especializadas } & \text { Incubadoras de sectores } \\
& \text { específicos }\end{array}$ \\
\hline \multirow{2}{*}{ Siglo XXI } & Incubadoras de nueva economía \\
\hline & Incubadoras virtuales \\
\hline
\end{tabular}

Fuente: Elaboración propia a partir de Fernández y Blanco (2011); Vaquero y Ferreiro (2015).

\section{Incubadoras: Conceptualización}

Después de una revisión de la literatura especializada, se aprecia que las conceptualizaciones existentes relativas a incubadora y vivero empresarial son diversas, ya que describen una serie de cualidades como: protección, seguridad, aceleración, entre otras (Hannon, 2004) que resultan coincidentes para ambos conceptos. A este respecto, diversos autores han realizado sus aportaciones (Ferreiro et al., 2018), como se muestra en la tabla 2 a continuación:

Tabla 2. Conceptualizaciones de incubadora y vivero

\begin{tabular}{|c|c|c|c|}
\hline Autor & Incubadoras & Autor & Viveros \\
\hline $\begin{array}{l}\text { Ciancio } \quad y \\
\text { Fardelli } \\
(2005)\end{array}$ & $\begin{array}{l}\text { Son organizaciones que acompañan y } \\
\text { aceleran el crecimiento de los proyectos } \\
\text { emprendedodors a través de asesorarmiento, } \\
\text { capacitación y asistencia financiera. }\end{array}$ & $\begin{array}{l}\text { Viadiu y } \\
\text { Martínez } \\
(2006)\end{array}$ & $\begin{array}{l}\text { Los viveros empresariales incentivan a la la } \\
\text { creación de nuevas empresas innovadoras y } \\
\text { que pueden estar ubicadas en sectores de } \\
\text { nuevas tecnologias. }\end{array}$ \\
\hline $\begin{array}{l}\text { Gozali, } \\
\text { Masrom, } \\
\text { Haron, y } \\
\text { Zagloel } \\
(2015)\end{array}$ & $\begin{array}{l}\text { Una incubadora de negocios es un edificio } \\
\text { que alberga empresas de inquilinos que } \\
\text { están en sus fases iniciales. }\end{array}$ & $\begin{array}{l}\text { Perdomo, } \\
\text { Arias y } \\
\text { Lozada } \\
\text { (2014) }\end{array}$ & $\begin{array}{l}\text { Son instalaciones donde se habilitan } \\
\text { temporalmente espacios para el } \\
\text { emprendimiento de actividades de I+D, } \\
\text { habitualmente se encuentran cercadealguna a a a } \\
\text { institución académica o centro de } \\
\text { investigación. }\end{array}$ \\
\hline $\begin{array}{l}\text { Leiva et al., } \\
\text { (2016) }\end{array}$ & $\begin{array}{l}\text { Una incubadora de empresas pretende } \\
\text { promover la creación, fortalecimiento y } \\
\text { desarrollo de nuevas empresas que pueden } \\
\text { por sí solas enfrentar las condiciones } \\
\text { competitivas del mercado. }\end{array}$ & $\begin{array}{l}\text { Blanco, } \\
\text { Oliva, } \\
\text { Bassa, y } \\
\text { Garcia } \\
(2016)\end{array}$ & 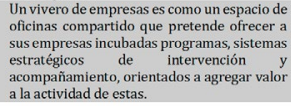 \\
\hline $\begin{array}{l}\text { Valenciano } \\
\text { y Uribe } \\
(2016)\end{array}$ & $\begin{array}{l}\text { Las incubadoras empresariales están } \\
\text { dotadas de un espacio destinado a su cesión } \\
\text { a nuevas empresas, fomentando la creación } \\
\text { y consolidación de estas, variando los } \\
\text { servicios que se prestan de un vivero a otro. }\end{array}$ & $\begin{array}{l}\text { Ferreiro } y \\
\text { Camino } \\
(2016)\end{array}$ & $\begin{array}{l}\text { Son entidades que proporcionan espacio } \\
\text { fisico y asistencia para la creación de nuevas } \\
\text { empresas y las ayudana a alcanzar un rápido } \\
\text { y exitoso desarrollo económico. }\end{array}$ \\
\hline $\begin{array}{l}\text { Ministerio } \\
\text { de } \\
\text { Economía, } \\
\text { Industria } y \\
\text { Competitiv } \\
\text { idad } \\
(2017)\end{array}$ & $\begin{array}{l}\text { Se entiende como incubadora a una } \\
\text { organización que da apoyo a los } \\
\text { emprendedores o inventores para que } \\
\text { transformen sus ideas en negocios de exito, } \\
\text { poniendo a su disposición una serie de } \\
\text { recursos y servicios. }\end{array}$ & $\begin{array}{l}\text { Valenciano } \\
\text { y Uribe } \\
(2016)\end{array}$ & $\begin{array}{l}\text { Entidades que estimulan el desarrollo local y } \\
\text { regional, facilitando la creación de nuevos } \\
\text { negocios con un sinfín de servicios. }\end{array}$ \\
\hline $\begin{array}{l}\text { Mipro } \\
\text { (2017) }\end{array}$ & $\begin{array}{l}\text { Se entiende por incubadora al espacio } \\
\text { diseñado para acelerar el crecimiento y } \\
\text { desarrollo de proyectos de emprendimiento } \\
\text { innovador que tengan relación con la } \\
\text { producción. }\end{array}$ & $\begin{array}{l}\text { Ferreiro et } \\
\text { al., (2018) }\end{array}$ & $\begin{array}{l}\text { Son instalaciones que albergan por un tiempo } \\
\text { ideas de negocio, estimulando la creación de } \\
\text { nuevas empresas innovadoras, brindando } \\
\text { varios servicios para la consecución de esta. }\end{array}$ \\
\hline
\end{tabular}

Fuente: Elaboración propia a partir de Ciancio y Fardelli (2005); Viadiu y Martínez (2006):Perdomo et al. (2014); Gozali, Masrom, Haron y Zagloel, (2015); Blanco, Oliva, Bassa y Garcia (2016) Ferreiro y Camino (2016); Leiva et al. (2016); Valenciano y Uribe (2016); Ministerio de Economía, Industria y Competitividad (2017); MIPRO (2017); Ferreiro et al., (2018)

Teniendo en cuenta lo previamente mencionado, en las diferentes regiones del mundo los conceptos antes expuestos resultan similares, sin importar el idioma en el que se exprese, las definiciones contemplan contenidos muy parecidos entre sí. Por ejemplo, en Reino Unido, el término incubadora o vivero mantiene el mismo significado. En Francia un vivero de proyecto empresarial es sinónimo de incubadora. En definitiva, los términos incubadora y vivero se pueden relacionar Valenciano y Uribe, 2016) Por ende, los servicios que ofrecen estos centros son varia- 
dos, desde locales a precios bajos; aportación al conocimiento, guía al plan de viabilidad del negocio y financiación (Valdés, 2015; Viadiu y Martínez, 2006), además de asistencias legales generales, procesos de producción (Elmansori, 2014), infraestructura operativa y administrativa a emprendedores (Risso et al., 2015), e intercambio de información con las demás empresas (Lacono y Nagano, 2014; Vaquero y Ferreiro, 2015); es decir, redes de contacto (Guerra et al., 2015).

Finalmente, Maculan et al. (2015) ponen de manifiesto que las incubadoras funcionan como un intermediario indispensable para reducir los costos de acceso a la información de naturaleza legal o técnica y a los recursos naturales, humanos y financieros, además permite la interacción con un mayor número de organizaciones y favorece a la creación de un capital intangible de conocimientos (p. 11).

\section{Clasificación de las incubadoras}

Las incubadoras empresariales han ido evolucionando desde su nacimiento según los requerimientos de los emprendedores y la nueva economía (Vaquero y Ferreiro, 2015; Blanco et al., 2018), así:

Primera generación, surgen en los años 80 y su actividad principal era rentar espacios físicos a nuevas empresas con servicios compartidos a un costo mínimo, además con el beneficio de la cercanía a instituciones y universidades, eso en la época en que los centros eran de uso mixto. Promover nuevos emprendedores para el desarrollo económico (Viadiu y Martínez, 2006).

Segunda generación, surgen en los 90 con el objetivo de complementar el espacio físico con servicios de asesoramiento, acceso a financiación y contactos con otras empresas. Se caracterizó por los vínculos directos con las universidades en la creación de nuevas empresas, motivando a la investigación de nuevas tecnologías, dando inicio al surgimiento de Empresas de Base Tecnológica (EBTs) en los Estados Unidos.

Tercera generación, se registra en 1998 donde su objetivo era proveer a los pequeños emprendedores de los recursos necesarios para la transferencia y exportación de sus productos innovadores a otros países; es decir, creando redes internacionales donde se puedan cooperar mutuamente en las diferentes actividades y poder transferir la tecnología, innovación y capacitaciones necesarias entre las incubadoras, donde los principales beneficiarios eran las empresas incubadas. Según Valenciano y Uribe (2016) los tipos de incubadoras pueden variar según el sector empresarial:

Incubadoras tecnológicas: Localizadas en parques tecnológicos que brindan apoyo especializado a empresas de base tecnológica enfocadas en la investigación, desarrollo e innovación (I+D+i).

Incubadoras Clásicas: Enfocadas al alojamiento de empresas Pymes, contribuyendo con infraestructura adecuada para su actividad inicial. Incubadoras industriales: Se crean nuevas empresas industriales más no se generan ideas emprendedoras, propiciando espacios físicos pertinentes, contribuyendo al crecimiento de la economía local.

Incubadoras microempresa: Incentivan a la creación de empresas en localidades de escasos recursos económicos, repercutiendo en su desarrollo a corto y largo plazo e impidiendo la inversión privada como consecuencia un alto índice de desempleo.

\section{Contribución de las incubadoras a la economía}

Las incubadoras han tenido un gran impacto en el desarrollo de la economía de cada región y país (Ferreiro et al., 2018), incentivando en la creación de nuevas empresas en los diferentes continentes (Leiva et al., 2016) permitiendo su supervivencia a través del tiempo y en la generación de fuentes de empleo (Ferreiro y Camino, 2016), además en la contribución fiscal de ambas partes, incrementando la riqueza nacional (Vaquero y Ferreiro, 2015).

Por otra parte, las incubadoras son fundamentales en generar lazos de cooperación con distintos emprendedores accediendo de esta manera a información y recurso de innovación (Valenciano y Uribe, 2016), poniendo a disposición de los emprendedores instrumentos necesarios para la aceleración de proyectos empresariales (Ferreiro et al., 2018). Un claro ejemplo son países europeos donde las incubadoras contribuyen a la economía mediante el incremento de la actividad productiva que se abre a nuevos mercados, permitiendo el desarrollo económico en las zonas donde radican, como del fortalecimiento de redes empresariales (Vaquero y Ferreiro 2015).

Finalmente, las pymes son un punto muy importante en la generación económica ya que han sido reconocidas como herramientas esenciales para la transformación en el país (Elmansori, 2014).

\section{Metodología}

La investigación planteada tiene carácter tanto cualitativo como cuantitativo ya que en primer lugar se ha realizado una revisión sistematizada de la literatura sobre la temática en estudio y se ha complementado con el uso de técnicas de análisis descriptivo y estadístico. Asimismo, el enfoque aplicado es de tipo exploratorio como descriptivo. Es exploratorio debido a que busca identificar a través de la revisión de artículos científicos, las variables principales que a su vez permitan establecer una comparación de la efectividad de la creación de incubadoras por países y regiones. Adicionalmente, conlleva un enfoque descriptivo, ya que tiene por objeto combinar criterios que permitan ordenar, sistematizar y dar a conocer un análisis de la influencia que ejercen las incubadoras en el desarrollo económico los países analizados.

Para el análisis empírico se hace uso de información estadística procedente de las siguientes bases de datos e informes: Anprotec, Sebrae, Senescyt, Ministerio de Industria y Productividad (MIPRO), Institución de Corfo en Chile y artículos científicos entre los más destacados Blanco, Ferreiro, Perdomo, Risso, Vaquero, entre otros. Desde el punto de vista espacial el estudio se centra en siete países Reino Unido, Alemania, España, México, Brasil, Chile y Ecuador, que fueron elegidos en base a un doble criterio: por un lado, disponibilidad estadística y, por otro lado, representan diferentes realidades sociales y económicas. En términos generales, el período analizado va del año 2013 al 2017. Por lo tanto, haciendo uso de las fuentes y datos anteriormente mencionados, se trata de identificar el comportamiento de la creación de incubadoras en los siete países a través de un análisis de varianzas con un ANOVA factorial.

\section{Resultados}

La creación de incubadoras empresariales como apoyo a nuevos emprendimientos ha generado un gran impacto en varios países del mundo. Por tanto, esta sección en primer lugar se analiza la homogeneidad de los datos a través de la prueba Levene y en segundo lugar se aplica ANOVA factorial para observar el comportamiento que existe entre los países al momento de crear las incubadoras.

En la tabla 3, se observa a los países seleccionados de Europa y Centro América con mayor número de incubadoras creadas, favorable a la dinámica de innovación y creación de nuevas formas o flujos económicos en beneficio de cada país (Valenciano y Uribe, 2016). En Sudamérica, dos de los tres países estudiados se han estancado en la creación de nuevas incubadoras (Guerra et al., 2015), excepto Brasil que presenta un crecimiento en la creación de estos centros como apoyo a emprendedores (Maculan et al., 2015).

Tabla 3. Número de incubadoras creadas

\begin{tabular}{lcccccc}
\hline \multicolumn{1}{c}{ Países } & $\begin{array}{c}\text { Año de Inicio } \\
\text { de }\end{array}$ & \multicolumn{5}{c}{ Número de incubadoras por año } \\
& Incubadoras & $\mathbf{2 0 1 3}$ & $\mathbf{2 0 1 4}$ & $\mathbf{2 0 1 5}$ & $\mathbf{2 0 1 6}$ & $\mathbf{2 0 1 7}$ \\
\hline Reino Unido & 1980 & 182 & 228 & 288 & 367 & 101 \\
Alemania & 1980 & 380 & 353 & 350 & 360 & 350 \\
España & 1980 & 303 & 353 & 578 & 227 & 402 \\
México & 1990 & 233 & 222 & 222 & 225 & 294 \\
Brasil & 1984 & 377 & 393 & 377 & 369 & 369 \\
Chile & 1990 & 22 & 14 & 16 & 23 & 18 \\
Ecuador & 1998 & 27 & 31 & 40 & 22 & 30 \\
\hline
\end{tabular}

Fuente: Elaboración propia a partir de Versino y Hoeser (2006); Amaral, Magalhães y Lima (2009); Mayer y Jiménez (2011); Corfo (2012); Ministerio de Economía (2012); Ferreiro et al., (2015); Senescyt (2015); Vaquero y Ferreiro (2015); Anprotec e Sebrae (2016); Bone, Allen y Haley(2017); InnovationsZentren (2017); Nivela, Vera y Gil (2017); Anprotec (2018); Blanco et al., (2018).

En la tabla 4, se denota que el porcentaje global de incubadoras creadas en los países estudiados es del 55\%, siendo Reino Unido uno de los referentes en Europa, donde por cada 100 incubadoras, se crean 63 centros y cierran 37. Es decir, los 63 centros creados son el resultado del promedio de incubadoras creadas de 2013 hasta 2017 
partido para el último valor obtenido para 2018, y estos multiplicados por 100 (ver fórmula), mientras que el porcentaje de incubadoras cerradas se obtiene de la diferencia. Por ende según Ciancio y Fardelli (2005) mayoritariamente Europa es un referente de apoyo a nuevos negocios a través de sus incubadoras con lo cual garantizan la supervivencia de los emprendimientos.

Respecto a la tasa de crecimiento en el quinquenio se describen los datos más relevantes: Reino Unido, evidencia un crecimiento constante excepto la fuerte caída del número de centros en el año 2017, razón por la que refleja una tasa de decrecimiento de $-11,78 \%$ para el periodo. México es el país que muestra mayor tasa de crecimiento de incubadoras, esto es el 4,65\%, que a criterio se puede decir que uno de los factores determinantes puede ser la ubicación fronteriza con Estados Unidos, país que forma parte de una de las economías más dinámicas del mundo. Para el caso de Ecuador la tasa de crecimiento es de $2,11 \%$, hay que considerar que en la última década, los gobiernos de turno han realizado un cambio sustancial a la matriz productiva y politicas públicas de apoyo al emprendimiento.

En la misma tabla se hace un analisis del porcentaje de creación y cierre, así como la tasa de crecimiento de las mismas, para lo cual se aplican las siguientes formulas:

\section{Tasa de creación:}

$$
\begin{aligned}
& \text { Dónde: } \quad T C=\frac{P u}{P t} * 100 \\
& \mathrm{Pu}=\text { Población Incubadora } \\
& \mathrm{Pt}=\text { Población total de incubadoras }
\end{aligned}
$$

Cabe indicar que esta fórmula sigue el trazado de una curva logística. Tasa de crecimiento:

$$
\begin{aligned}
& \text { Donde: } T C=\operatorname{Ln}\left[\frac{P f}{P b}\right] *\left[\frac{1}{P}\right] * 100 \\
& \text { Ln = Logaritmo natura. } \\
& \mathrm{Pf}=\text { Población final } \\
& \mathrm{Pb}=\text { Población base } \\
& \mathrm{P}=\text { Periodo }
\end{aligned}
$$

\begin{tabular}{|c|c|c|c|c|c|c|c|c|c|c|}
\hline \multirow{2}{*}{ Países } & \multicolumn{5}{|c|}{ Años } & \multirow{2}{*}{$\begin{array}{c}\text { Promedio } \\
\text { 2013-2017 }\end{array}$} & \multirow{2}{*}{$\begin{array}{c}\text { Enero d } \\
2018\end{array}$} & \multirow{2}{*}{$\begin{array}{c}\% \text { de } \\
\text { creación de } \\
\text { incubadora }\end{array}$} & \multirow{2}{*}{$\begin{array}{c}\% \text { de } \\
\text { cierre de } \\
\text { incubadora }\end{array}$} & \multirow{2}{*}{$\begin{array}{c}\begin{array}{c}\text { Tasa de } \\
\text { crecimiento } \\
(\%)\end{array} \\
(\%)\end{array}$} \\
\hline & 2013 & 2014 & 2015 & 2016 & 2017 & & & & & \\
\hline Reino Unido & 182 & 228 & 288 & 367 & 101 & 233 & 368 & 63 & 37 & $-11,78$ \\
\hline Alemania & 380 & 353 & 350 & 360 & 350 & 359 & 603 & 59 & 41 & $-1,64$ \\
\hline España & 353 & 465 & 578 & 227 & 402 & 405 & 656 & 62 & 38 & 2,60 \\
\hline México & 233 & 222 & 222 & 225 & 294 & 239 & 487 & 49 & 51 & 4,65 \\
\hline Brasil & 377 & 393 & 377 & 369 & 369 & 377 & 673 & 56 & 44 & $-0,43$ \\
\hline Chile & 22 & 14 & 16 & 23 & 18 & 19 & 120 & 16 & 85 & $-4,01$ \\
\hline Ecuador & 27 & 31 & 40 & 22 & 30 & 30 & 120 & 25 & 75 & 2,11 \\
\hline Total General & 1.574 & 1.706 & 1.871 & 1.593 & 1.564 & 1.662 & 3.027 & 55 & 45 & \\
\hline
\end{tabular}

Tabla 4. Comparativo de las incubadoras empresariales Periodo 2013-2017

Fuente: Elaboración propia a partir de Versino y Hoeser (2006); Amaral, Magalhães y Lima (2009); Mayer y Jiménez (2011); Corfo (2012); Ministerio de Economía (2012); Ferreiro et al., (2015); Senescyt (2015): Vaquero y Ferreiro (2015); Anprotec e Sebrae (2016); Bone, Allen y Haley(2017);

InnovationsZentren (2017); Nivela, Vera y Gil (2017); Anprotec (2018); Blanco et al., (2018).

Para confirmar lo mencionado en las tablas 3 y 4 se aplica la técnica ANOVA, que mide el comportamiento de la creación de incubadoras a través del modelo factorial simple con efectos fijos, cuya ecuación es:

$$
\mathrm{X}_{\mathrm{ij}}=\mu+\mathrm{A}_{\mathrm{j}}+\varepsilon_{\mathrm{ij}}
$$

Como se ha explicado previamente, las variables a tener en cuenta son las siguientes:

Variable independiente: país, con cinco niveles.

Variable dependiente: número de incubadoras registradas.

La hipótesis planteada es:

$\mathrm{H}_{0}$ : Existen diferencias significativas entre los siete países al momento de la creación de incubadoras.

$\mathrm{H}_{1}$ : No existen diferencias significativas entre los siete países al momento de la creación de incubadoras.

$$
\begin{aligned}
& \text { Ho } \mu_{1}=\mu_{2}=\mu_{3}=\mu_{4} \\
& \mathrm{H}_{1}: \mu_{1} \neq \mu_{2} \neq \mu_{3} \neq \mu_{4}
\end{aligned}
$$

Estadístico de contraste: será una F de Snedecor:

$$
\mathrm{F}_{(\mathrm{k}-1),(\mathrm{n}-\mathrm{k}) ;(1-\alpha)}=\frac{\hat{\mathrm{S}}_{\mathrm{F}}^{2}}{\hat{\mathrm{S}}_{\mathrm{E}}^{2}}
$$

Contraste unilateral: A mayor numerador respecto al denominador mayor posibilidad de rechazar la hipótesis nula. Mientras más pequeño, mayor posibilidad de no rechazarla.

En la tabla 5, la prueba de Levene para la igualdad de varianzas confirma que, en este estudio comparativo de creación de incubadoras por países, existe homogeneidad en los datos en los años de estudio, con un nivel de significancia $p=0,001$. Lo anterior corrobora que las tendencias de las políticas actuales están orientadas hacia el apoyo de nuevos emprendimientos (Valdés, 2015).

Tabla 5. Prueba de homogeneidad de varianzas

\begin{tabular}{|c|c|c|c|c|c|}
\hline & $\begin{array}{c}\begin{array}{c}\text { Suma de } \\
\text { cuadrados }\end{array} \\
\text { cols }\end{array}$ & G1 & Media cuadrática & $F$ & sig. \\
\hline Inter-grupos & $716^{\prime} 069.486$ & 6 & $119^{\prime} 344.914$ & 28.906 & 0,000 \\
\hline Intra-grupos & $115^{\prime} 603.200$ & 28 & $4^{1} 128.686$ & & \\
\hline Total & $831^{\prime} 672.686$ & 34 & & & \\
\hline
\end{tabular}
Periodo 2013-2017

\begin{tabular}{rccc}
\hline Estadístico de Levene & gl1 & gl2 & Sig. \\
\hline 5.023 & 6 & 28 & 0,001 \\
\hline
\end{tabular}

Fuente: Elaboración propia a partir de Versino y Hoeser (2006); Amaral, Magalhães y Lima (2009); Mayer y Jiménez (2011): Corfo (2012): Ministerio de Economía (2012): Ferreiro et al., (2015); Senescyt (2015); Vaquero y Ferreiro (2015); Anprotec e Sebrae (2016); Bone, Allen y Haley(2017);

InnovationsZentren (2017); Nivela, Vera y Gil (2017); Anprotec (2018); Blanco et al., (2018).

La aplicación de ANOVA, en la tabla 6, muestra que efectivamente existen diferencias significativas en el comportamiento al momento de crear las incubadoras, en los países estudiados. Esto se evidencia por tener una significancia de $\mathrm{p}=0,000$, por tanto, se acepta la hipótesis nula.

Fuente: Elaboración propia a partir de Versino y Hoeser (2006); Amaral, Magalhães y Lima (2009); Mayer y Jiménez (2011): Corfo (2012): Ministerio de Economía (2012); Ferreiro et al., (2015); Senescyt (2015): Vaquero y Ferreiro (2015): Anprotec e Sebrae (2016); Bone, Allen y Haley(2017); InnovationsZentren (2017); Nivela, Vera y Gil (2017); Anprotec (2018); Blanco et al., (2018).

Teniendo como base los resultados de la tabla anterior, respecto a la existencia de diferencias significativas entre los países de estudio, se aplica ANOVA factorial, para identificar qué países son los que causan esta diferencia.

En la tabla 7 se muestran los resultados que genera ANOVA factorial y

\begin{tabular}{|c|c|c|c|c|}
\hline \multirow{2}{*}{ País } & \multirow{2}{*}{$\mathbf{N}$} & \multicolumn{3}{|c|}{ Subconjunto para alfa $=, 05$} \\
\hline & & 1 & 2 & 3 \\
\hline Chile & 5 & 18,60 & & \\
\hline Ecuador & 5 & 30,00 & & \\
\hline Reino Unido & 5 & & 233,20 & \\
\hline México & 5 & & 239,20 & \\
\hline Alemania & 5 & & 358,60 & 358,60 \\
\hline España & 5 & & & 372,60 \\
\hline Brasil & 5 & & & 377,00 \\
\hline Sig. & & 1,000 & 0,061 & 0,999 \\
\hline
\end{tabular}
muestra la agrupación por países de iguales condiciones, bajo el criterio de similares comportamientos al momento de crear las incubadoras. Por un lado, los países del grupo 1: Chile y Ecuador presentan un comportamiento similar en el surgimiento de incubadoras, quizá por la similitud en sus políticas públicas, efectividad de servicios, calidad, entre otras. Por otro lado, el grupo 2 de países conformados por Reino Unido, México y Alemania mantienen un comportamiento semejante en cuanto a la generación de incubadoras, en este grupo, es importante la presencia de México junto a dos países europeos. Finalmente, el tercer grupo lo conforman España, Brasil y Alemania, siendo este último parte del grupo 2 y 3.

Fuente: Elaboración propia a partir de Versino y Hoeser (2006); Amaral, Magalhães y Lima (2009); Mayer y Jiménez (2011); Corfo (2012); Ministerio de Economía (2012); Ferreiro et al., (2015); Senescyt (2015); Vaquero y Ferreiro (2015); Anprotec e Sebrae (2016); Bone, Allen y Haley(2017); InnovationsZentren (2017); Nivela, Vera y Gil (2017); Anprotec (2018); Blanco et al., (2018).

\section{Conclusiones}

En el presente trabajo se observa que el emprendimiento es parte fundamental en el desarrollo económico de los países, a través de la generación de nuevos empleos, recaudación de impuestos e incentivo para la inversión extranjera. 
Para brindar apoyo y acompañamiento a nuevos emprendimientos en el proceso de pre-incubación, incubación y post-incubación de nuevas empresas, nacen las incubadoras, cabe destacar que en la actualidad estos centros constituyen un elemento clave y representativo en el mundo, debido a que coadyuvan a la supervivencia del tejido empresarial.

Este estudio aplica pruebas estadísticas con el objetivo de conocer posibles similitudes en el comportamiento al momento de la creación de las incubadoras, con ANOVA se mide el comportamiento de la creación de incubadoras, observando la existencia de diferencias entre los siete países analizados, sin embargo, se hace necesario conocer que países motivan tales diferencias, para lo cual se aplica ANOVA factorial, a través del cual se obtiene la conformación de tres grupos de países que tienen similitud de comportamiento en la generación de incubadoras, esto puede darse por la igualdad de características de funcionamiento de acuerdo con su territorio económico.

Chile y Ecuador conforman el grupo 1, estos mantienen una similitud en el surgimiento de Incubadoras debido a sus políticas de desarrollo, que estimulan proyectos emprendedores, como alternativa para potenciar el crecimiento económico del país (Guerra et al., 2015).

Por su parte Reino Unido, México y Alemania, conforman el grupo 2. En el caso de los países europeos, generan entornos favorables para el emprendedor dado a la posición estable de su política y economía que les permite a los emprendimientos incursionar en mercados internacionales. En cuanto a México, cabe destacar que su posición geográfica y relaciones comerciales transfronterizas con una de las principales economías del mundo, le permite tener similitudes con los citados países europeos y destacar en el desarrollo de sus emprendimientos (Vargas, 2014; Bone et al., 2017).

El grupo 3 está conformado por España, Brasil y Alemania, la similitud en el comportamiento al momento de crear las incubadoras puede estar representada por la dinámica de su economía al mantener significativas alianzas comerciales (Valenciano y Uribe, 2016; Maculan et al., 2015).

Cabe recalcar que a un nivel de significancia menor del $5 \%$, se acepta la hipótesis nula, es decir, que hay diferencias significativas entre los países europeos y latinoamericanos al momento de la creación de incubadoras, esto se debe a que Reino Unido, Alemania y España son países desarrollados y tienen políticas y programas orientados al impulso del emprendimiento a diferencia del resto de países Latinoamericanos, sin embargo, cabe recalcar que México y Brasil son el referente de centro y el Sur de América. En la última década los países latinoamericanos mejoran sus matrices productivas y consecuentemente sus políticas públicas para el apoyo al emprendimiento, valorado como eje del crecimiento económico. Finalmente, se recomienda retomar esta investigación como futuras líneas de investigación, las relacionadas con el análisis de los factores internos y externos que determinan el fracaso o éxito de las incubadoras.

\section{Referencias}

Alba, C. (2015). Modelo de Incubación de Empresas: Una Propuesta. Perspectivas(36), 65-90. Obtenido de http://www.redalyc.org/html/4259/425943146003/ Amaral, M., Magalhães, L., \& Lima, M. (2009). Uma proposta de avaliação de maturidade em parques cientificos, techológicos e de inovação-PCTIS. Locus Cientifico, 3(4),
$115-122$. Obtenido de http://Www.anprotec.org.br/ArquivosDin/locus_cientifico_V3N4_laminas_pdf__O2.pdf

Anprotec. (23 de Febrero de 2018). Asociación Nacional de Entidades Promotoras de Emprendedores Inovadores. Obtenido de http://anprotec.org.br/site/2018/02/ toras de Emprendedores Inovadores.
cerne-e-apresentado-como-modelo-de-referencia-no-world-incubation-summit-2018/
Anprotec e Sebrae. (2016) Estudo de impacto económico segmento de incubadoras de empresas do Brasil. Estudos, 1-36. Obtenido de http://anprotec.org.br/site/publicacoes-anprotec/estudos-e-pesquisas

coes-anproteclestudos-e-pesquisas/
Blanco, F., Oliva, M., Bassa, J., \& Garcia, C. (2016). Los servicios que prestan los viveros de empresas en España. Ranking 2015. Funcas, 1-62. empresas en España. Ranking 2015. Funcis, 1-62.
Blanco, J., Garcia, C., Fernandez, M., Oliva, M., Bassa, J., \& Vaisman, A. (2018). Los servicios que prestan los viveros de empresas en España Ranking 2016/2017. Funcas, 1-80. Obtenido de http:///cincubator.com/wp-content/uploads/2017/05Niveros2017.pdf Bone, J., Allen, O., \& Haley, C. (2017). Business incubators and accelerators: The national picture. Departamento de Negocios, Energía y Estrategia Industrial, 1-76. Obtenido de https://www. gov.uk/government/publications/business-incubators-and-accelerators-the-national-picturemhistory

Boza, J., Tachong, L., \& Moreira, M. (2016). La Universidad como catalizador del desarrollo local en Ecuador. Revista Caribeña de Ciencias Sociales, 1-13. Obtenido de http:// www.eumed.net/rev/caribe/2016/06/universidad. $h$ tm Ciancio, M., \& Fardelli, C. (2005). Incubadoras de empresas en Argentina: Surgimiento, desarrollo y perspectivas. (págs. 1-16). Argentina: Altec. Obtenido de http://www.
littec. ungs.edu.ar/pdfespa\%F $10 / 1 / D T \% 2002-2005 \% 20$ Fardelli-Ciancio.pdf

Corfo. (6 de Marzo de 2012). Unidad de Monitoreo y Evaluación Gerencia de Estrategia y Estudio CORFO. Obtenido de file:///C:/Users/empire/Downloads/Reporte+de+indcadores+de+incubados+2012,3.pd

Da Silva, A., Furtado, J., \& Zanini, R. (2015). Evolução do empreendedorismo no brasil baseada nos indicadores do global entrepreneurship monitor (GEM). Revista Produção, 15(2), 758-780. doi:https:///doi.org/10.14488/1676-1901.v15i2.1940 De Sousa, D, Goncalves, R., Almeida, M. \& Sacomano, J. (2017). Parques
Tecnológicos e incubadoras: Uma análise do processo de pré-incubacao de empresas de base tecnológica. Interciencia, 42(5), 313-319. Obtenido de https://search.proquestcom/ docview/1955969528/fulltextPDF/4D5D059D348C4EOEPQ/1?accountid=36765

Elmansori, E. (2014). Business incubators in the arab world: Comparative study of Jordan and UAE business incubators. World Journal of Science, Technology and Sustainable Development, 282-293. Obtenido de https:///search.proqu

Fernández, F., \& Blanco, F. (2011). Incubación de empresas y Soft Landing empresarial en los países socios mediterráneos. Economía y competitividad en el área Euro mediterránea ICE, 161-174. Obtenido de http://Www.revistasice.com/CachePDF/CE 861 161-176 437C543A141EC25ACCE546901FC1D088.pdf

M. (2015). Analysis of business incubators in Galicia through the «Integral model of economic profitabilitys. Investigaciones Regionales, 33 7-31. Obtenido de $h$ ttps
$P Q / 2$ ?accountid $=36765$

Ferreiro, F. \& Camino M. (2016). Análisis cuantitativo de los Centros de iniciativas empresariales medido a través del método de la balanza fiscal: El caso Gallego. Revista Galega (1) Ferreiro, F., Mendo empresas españolas en el mercado de trabajo. Contaduría y Admíst doi:https://doi.org/10.22201/fca.24488410e.2018.926

Gonzalez. D. (2017). Desarrollo de las incubadoras de empresas en Perú y Políticas Públicas de promoción: Un estudio de caso. ALTEC, 1-16. Obtenido de http://Www.uam.mx Publicas de promoción: Un estudio de caso.
altec2017/pdfs/ALTEC 2017 paper $403 . p d f$

Gozali, L Masrom, M. Haron H. \& Zagloel, T. (2015). A framework of successful E-business incubator for indonesian public universities. The Asian Journal of Technology ManE-business incubator for indonesian public universities.
agement, 8(2), 118-131. doi:10.12695/aitm. 2015.8.2.4

Guerra, O., Hernández, D., \& Triviño, C. (2015). Incubadora de empresas: Vía para el emprendimiento en las universidades. Revista Universidad y Sociedad, 7(2), 110-114. Obt\&t/ng=en Hannon, P. (2004). A Qualitative Sense-Making Classification of Business Incubation Environments. DeepDyve, 7(4), 274-283. Obtenido de https://www.deepdyve com/lo/emerald-publishing/a-qualitative-sense-making-classification-of-business-incuba-

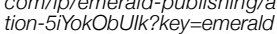

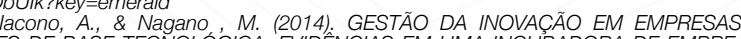
NASCENTES DE BASE TECNOLÓGICA: EVIDENCIAS EM UMA INCUBADORA DE EMPRESAS NO BRASIL. Interciencia, 39(5), 296-306. Obtenido de https://search.proquest.com/ docview/1535664207/fulltextPDF/3017A5A13C4149A1PQ/1? ?accountid=36765 InnovationsZentren. (2017). Recuperado el 26 de Julio de 2018, de Bundesverbandes Deutscher Innovations-Technologie- und Gründerzentren e.V.: https://Www.innovationszentren. de/39-0-Downloads. html

Lahorgue, M. (09 de Abril de 2010). Sistema de indicadores de innovación: el caso de las incubadoras de empresas en Brasil. Obtenido de In $N$ Congreso Iberoamericano de indicadores de Ciencia y Tecnología. Nuevas demandas sociales y tendencias en información cientifica y tecnológica: In IV Congreso Iberoamericano de Indicadores de Ciencia y Tecnología. Nuevas demandas sociales y tendencias en información cientifica y tecnológica Leiva, J., Monge, R., \& Morales, C. (2016). Empresas incubadas por el Centro de Incubación de Empresas del TEC: Un análisis cualitativo. Investiga. TEC(26), 15-16. Obtenido de $h$ ttp://revistas.tec.ac.cr/index.php/investiga_tec/artic/l/view/2564/2349

Maculan, A., Jiménez C., \& Castellanos, O. (2015). Aprendizaje en el proceso de in cubación de empresas de base tecnológica. Económicas CUC, 36(1), 15-48. doi:http://dx.dol org/10.17981/econcuc.36.1.2015.21

Mayer, E., \& Jiménez, K. (2011). Las incubadoras de negocios en México: un análisis descriptivo.
pdf/4419/441942926007.pdf

Messina, M., \& Hochsztain, E. (2015). Factores de éxito de un emprendimiento: Un estudio exploratorio con base en Técnicas de Data Mining. TEC Empresarial, 9(1), 31Obtenido de hittp://revistas.tec.ac.cr/index.php/tec_empresarial/article/vieW/2206/2006 7 de Julio de 2018, de Mapa de las incubadoras de negocios en Chile: http://Www.innovacion. gob. cl/2012/06/mapa-de-las-incubadoras-de-negocios-en-chile/

Ministerio de Economía, Industria y Competitividad. (2017). Evolución del comercio exterior por característica de la empresa. España: Boletín económico de ICE. Recuperado el 14 de Junio de 2018, de https://ebookcentral.proquest.com/lib/utasp/reader.action?doclD $=4869944$ \&query=incubadoras+de+negocios+en+america

MIPRO. (16 de Agosto de 2017). Ministerio de Industria y Productividad. Recuperado el 14 de Junio de 2018, de Mipro iniciará incubadora de empresas: https://Www.industrias. gob.ec/mipro-iniciara-incubadora-de-empresas/

Nivela, J., Vera, F., \& Gill, $R$. (2017). Incubadora de negocios, innovación del fu-
turo económico. Ciencia e investigación,. 2(8). 33-36. doi:https://doi.org/10.26910 issn.2528-8083vol2iss8.2017pp33-36

Perdomo, G., Arias, J., \& Lozada, N. (2014). Business incubator research a review and future directions. Pensamiento y Gestión(37), 41-65. doi:http://dx.doi.org/10.14482 pege.37.7020

Perdomo, G., Arias, J., \& Lozada, N. (2016). Análisis comparativo del cambio or-

ganizacional e institucional de dos incubadoras de empresas. Semestre económico, 19(39), Risso, M., Diaz, L., \& Ramassotti, E. (2015). Proyecto de incubación de pequeñas unidades productivas enmarcadas en los valores de la Economía Social y Solidaria. Ingenieria Solidaria, 11(18), 87-95. doi:https://doi. org/10.16925/in.v11i18.994

Senescyt. (9 de Marzo de 2015). Secretaria de Educación Superior, Ciencia, Tecnología e Innovación. Recuperado el 14 de Junio de 2018, de Emprendedores del Ecuado invierten su talento en el Banco de ldeas: https://www.educacionsuperior.gob.ec/emprendedores-del-ecuador-invierten-su-talento-en-el-banco-de-ideas/

SENESCYT. (2017). Secretaría de Educación Superior, Ciencia, Tecnología e Innovación. Recuperado el 14 de Junio de 2018, de Documentos de interés Ensamble 2017: http:// programasbecas. educacionsuperior.gob.ec/documentos-de-interes-ensamble-2017/

dimiento lasallep.edu.mX/XIHMAl/index. php/xihmai/article/view/248/221

Valenciano, I. \& Uribe, J. (2016). Viveros empresariales y desarrollo local. Experiencia del sur de Europa (Andalucía, España). Revista de Ciencias Sociales (Ve), XXII(1), 73-84. encia del sur de Europa (Andalucia, Espana). Revista de Ciencias
Obtenido de http://www.redalyc. org/articulo.oa?id=28046365006

de http://Www. redalyc.org/articulo. oa?id $=28046365006$
Vaquero, G., \& Ferreiro, F. (2015). Experiencias regionales en viveros de empresas. Revista de estudios regionales(102), 177-208. Obtenido de http://www.redalyc.org/articulo. Revista de estudios
oa?id $=75539637006$

Vargas, J. (2014). Incubadoras de empresas como aliadas en la reducción de costos de transacción de los emprendedores en México. Reuna, 19(5), 5-14. Obtenido de http: revistas. una.br/index.php/reuna/article/view/678

Versino, M., \& Hoeser, U. (2006). A diez años del inicio de la incubación de "Empresas de base tecnológica" en Argentina: Balance de la evolución del fenómeno y análisisde experiencias recientes. Redes, 12(24), 15-41. Obtenido de file:///C:/Users/empire/Downloads/51d5b630c8b62.pdt

Viadiu, M., \& Martínez, A. (2006). Tipologías de viveros de empresas: Tradicionales y especializadas. Investigaciones Europeas de Dirección y Economía de la Empresa, 12(1), 133-

152. Obtenido de $h$ ttp://www.redalyc.org/articulo.oa?id $=274120878006$ 